\title{
Transperineal endoscopic drainage of a presacral and paraspinal abscess
}
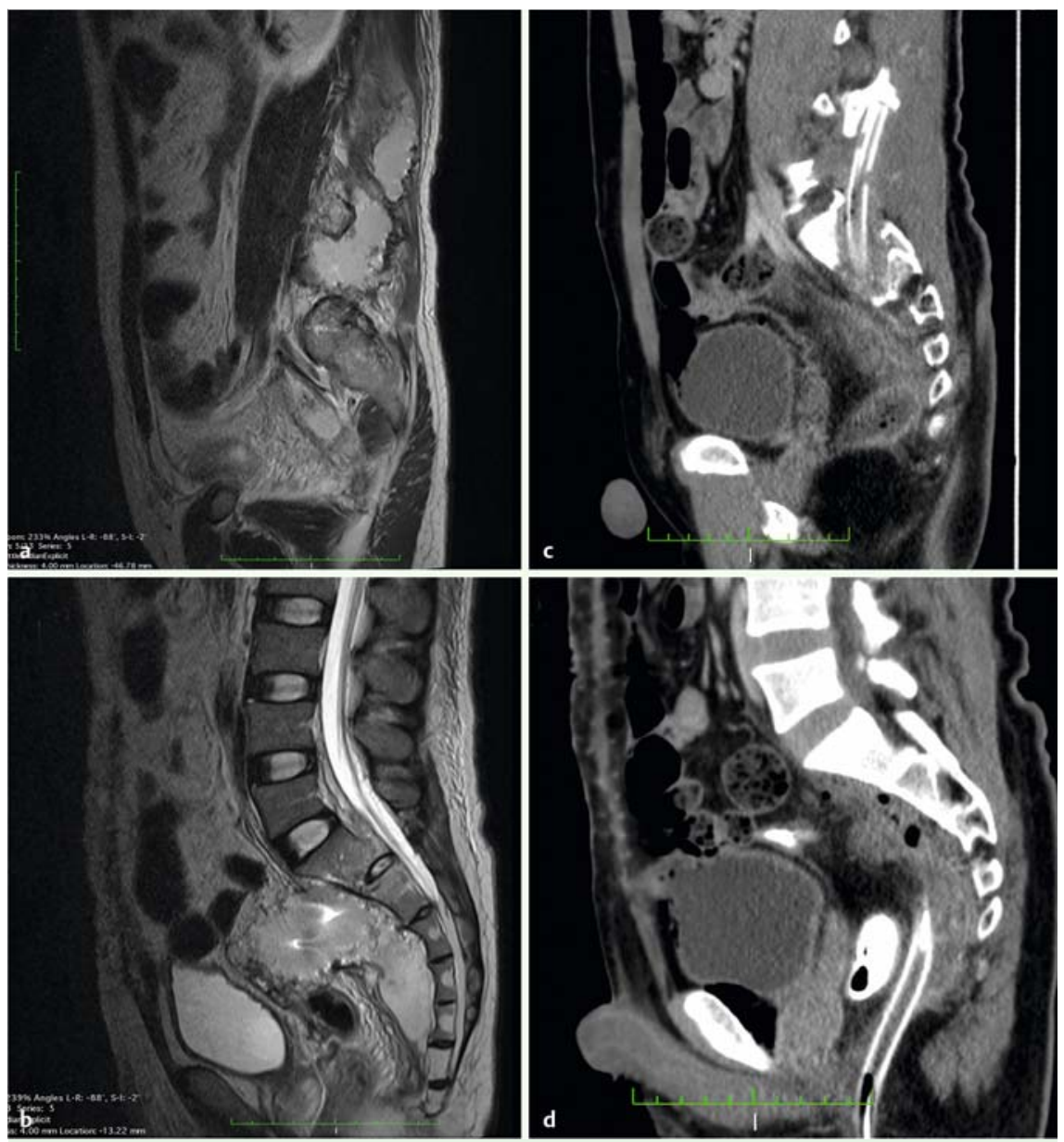

Fig. 1 Imaging 10 days after the penetrating perineal injury showing: a, b on magnetic resonance imaging (MRI), a presacral abscess with paraspinal extension; $\mathbf{c}, \mathbf{d}$ on a contrast enhanced computed tomography (CT) scan after the drainage procedure, a reduction in the size of the abscess and the drainage catheter in situ.

Image-guided percutaneous catheter drainage (PCD) has largely replaced open drainage for deep body-cavity abscesses in the era of interventional radiology [1]; however, presacral abscesses are often not easily accessible.

A healthy 36-year-old man fell from a height of 3 meters while working in a construction yard. His buttock landed on a steel rod resulting in penetrating trauma to the perineum. The rod, which was $2 \mathrm{~cm}$ in diameter and used for reinforcing concrete, entered his body to a depth of at least of $30 \mathrm{~cm}$. Despite this, the patient remained stable and was able to remove the rod himself before seeking medical help.
On arrival at the hospital, examination revealed a $2-\mathrm{cm}$ perineal laceration with air gushing out from it $4 \mathrm{~cm}$ from the anus in the three o'clock position. Digital examination of the rectum showed a rectal wall defect. A computed tomography (CT) scan of the abdomen and pelvis showed a comminuted fracture of the vertebral bodies of L5 to S2, paravertebral and pelvic hematomas, and a pneumoperitoneum. A laparotomy was performed. Apart from the rectal perforation, there was a $3-\mathrm{cm}$ perforation of the sigmoid colon, which was repaired primarily. The rectal perforation, being extraperitoneal, was left untouched. A diversion sigmoid colostomy was fashioned.
On postoperative day 10 , the patient was noted to have a swinging fever and a leukocytosis. Reassessment CT and a magnetic resonance imaging (MRI) scan showed a presacral abscess, measuring $10.2 \times 7.0 \times$ $4.6 \mathrm{~cm}$ in size, with paraspinal extension of $12.4 \times 3.9 \times 2.6 \mathrm{~cm}$ ( $\bullet$ Fig. $1 \mathrm{a}$ ). Rectal contrast injection showed extravasation of contrast but the perforation was not communicating with the abscess cavity. Drainage of the presacral abscess was performed on postoperative day 14 . The perineal wound, which was by then $2 \mathrm{~cm}$, was digitally dilated. A 12-mm laparoscopic port was inserted and carbon dioxide was insufflated. A track was created by blunt dissection using a $10-\mathrm{mm} 30^{\circ}$ laparoscope. As the scope probed into the abscess cavity, there was giving way and drainage of frank pus occurred. A gastroscope (Olympus, Japan) was used to aspirate pus and to perform irrigation ( Fig. 2). A 28-Fr chest drain was then inserted over a guidewire ( Fig. 3; $\bullet$ Video 1 ).

A further reassessment scan showed marked interval reduction in the size of the collection ( $\bullet$ Fig. $1 \mathbf{b}$ ). Culture of the pus yielded Escherichia coli and Enterobacter cloacae. Irrigation with $100 \mathrm{~mL}$ of saline was performed twice a day via the drain. A repeat endoscopic drainage was performed 2 weeks later and the patient remained afebrile after the second endoscopic drainage.

To perform PCD, which can be transabdominal, transperineal, or transgluteal, one needs to find a straight path that does not traverse anatomical structures such as the sacrum, rectum, and small bowel [2]. Transrectal drainage with endorectal ultrasound guidance is another option that can be used, especially when the collection is related to recent rectal surgery [3]. These are non-invasive interventions, but they are limited to the placement of relatively small drainage catheters, usually $8-10 \mathrm{Fr}$.

In this case, endoscopic drainage not only allowed drainage of pus but also deloculation and removal of solid residuals. Insertion of a large-bore drainage catheter was possible, with use of the track created, permitting repeated endoscopic lavage and debridement. This is the first case in the literature to report the endoscopic drainage of a presacral abscess. A similar technique has been described in the management of necrotizing pancreatitis $[4,5]$.

\section{Endoscopy_UCTN_Code_TTT_1AT_2AF}

Competing interests: None 


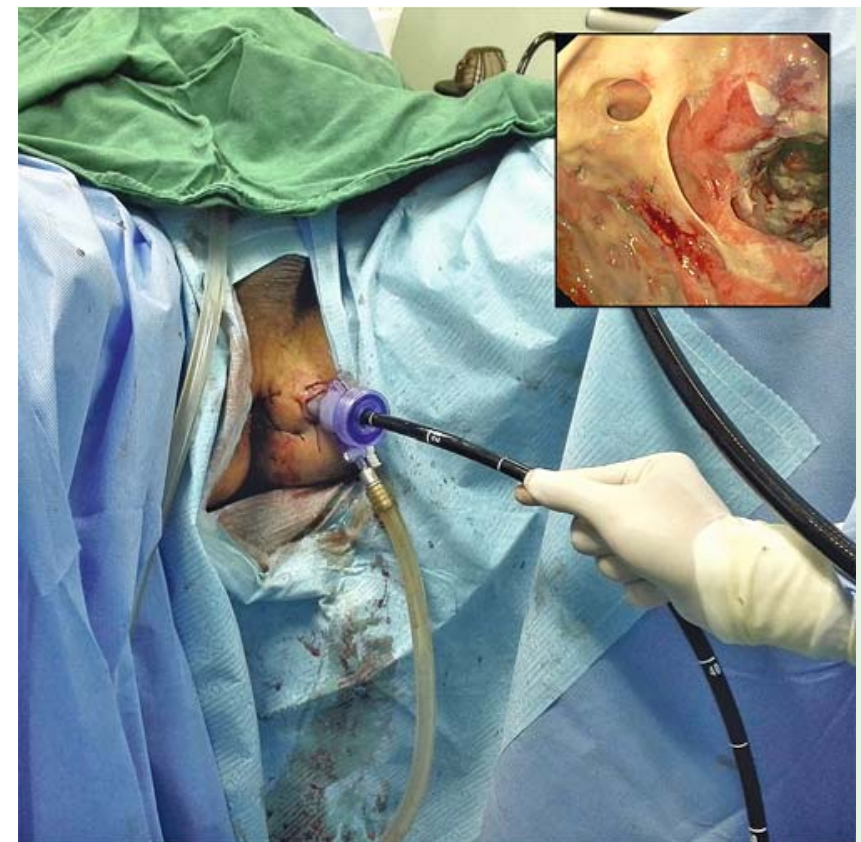

Fig.2 Transperineal endoscopic drainage of the presacral and paraspinal abscess. The photo at the top right-hand corner shows an endoscopic view of the abscess cavity.
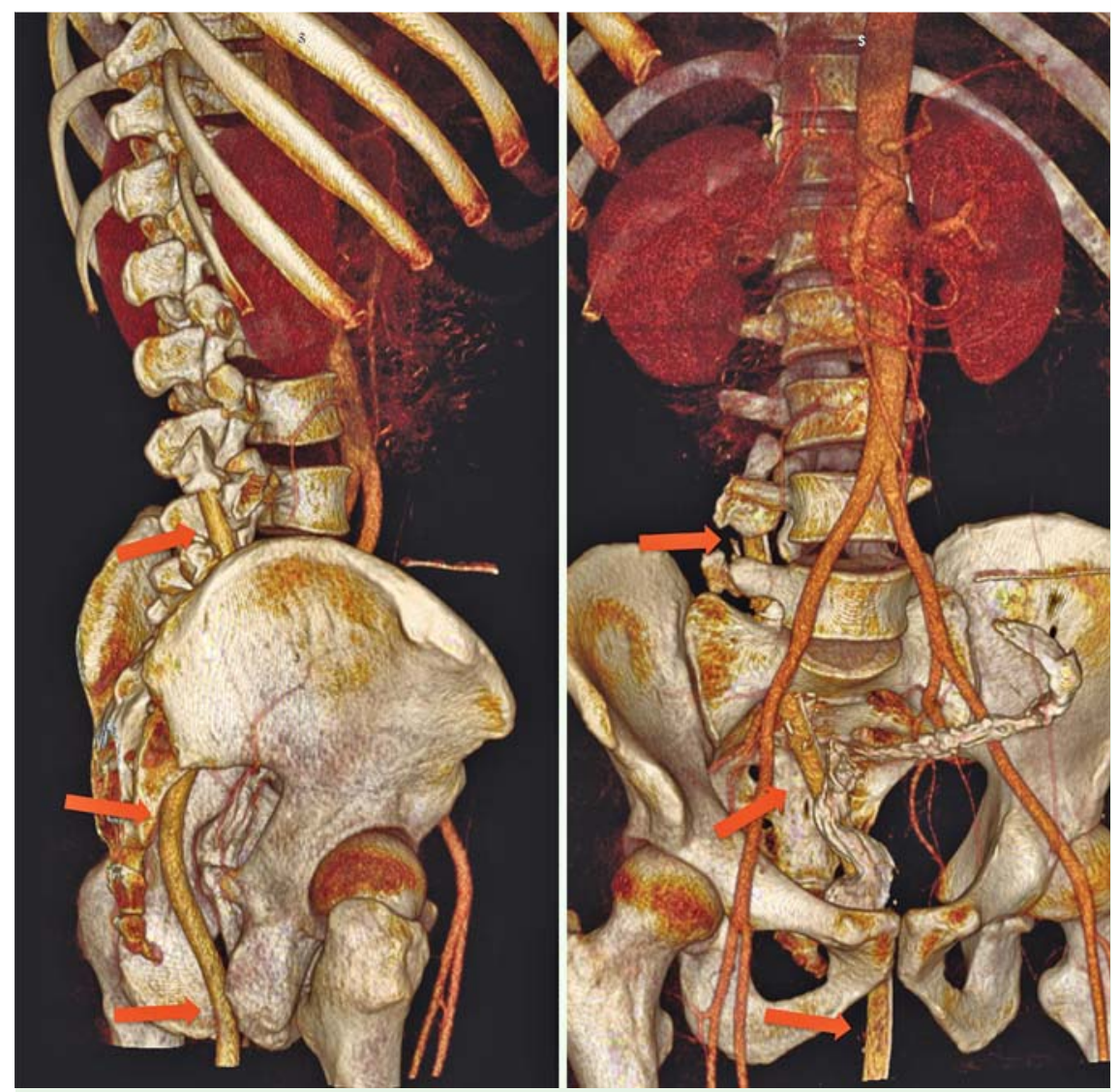

Fig. 3 Three-dimensional reconstruction of the post-drainage computed tomography (CT) scan showing the position of the drainage catheter (red arrows). Note the rectum is vaguely seen because of the presence of rectal contrast.

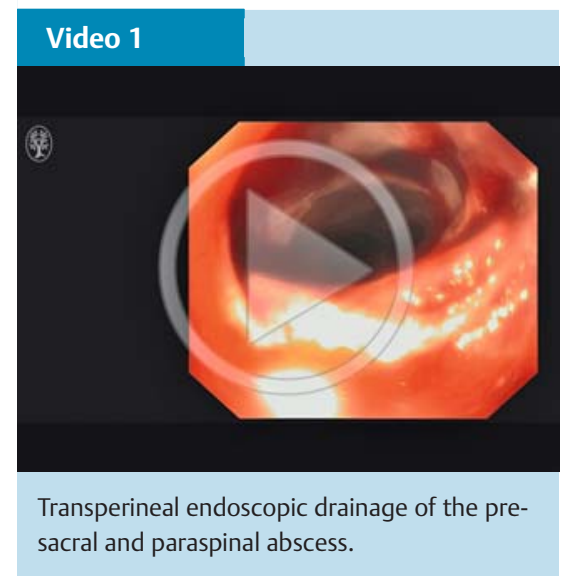

\section{Chi Chung Foo', ${ }^{1,2}$, Zhong Hui Liu², Joe King Man Fan ${ }^{1,2}$}

1 Division of Colorectal Surgery, Department of Surgery, The University of Hong Kong, Hong Kong, China

2 Department of Surgery, The University of Hong Kong - Shenzhen Hospital, Shenzhen, China

\section{References}

1 Lorenz JM, Al-Refaie WB, Cash BD et al. ACR appropriateness criteria radiologic management of infected fluid collections. J Am Coll Radiol 2015; 12: 791 - 799

2 Trambert JJ. Percutaneous interventions in the presacral space: CT-guided precoccygeal approach - early experience. Radiology 1999; 213: $901-904$

3 Puri R, Choudhary NS, Kotecha $\mathrm{H}$ et al. Endoscopic ultrasound-guided pelvic and prostatic abscess drainage: experience in 30 patients. Ind J Gastroenterol 2014; 33: 410413

4 Becker $V$, Huber $W$, Meining $A$ et al. Infected necrosis in severe pancreatitis - combined nonsurgical multi-drainage with directed transabdominal high-volume lavage in critically ill patients. Pancreatology 2009; 9: 280-286

5 Yamamoto $N$, Isayama $\mathrm{H}$, Takahara $\mathrm{N}$ et al. Percutaneous direct-endoscopic necrosectomy for walled-off pancreatic necrosis. Endoscopy 2013; 45 (Suppl. 02): E44-E45

\section{Bibliography}

DOI http://dx.doi.org/

10.1055/s-0042-118597

Endoscopy 2016; 48: E361-E362

(C) Georg Thieme Verlag KG

Stuttgart · New York

ISSN 0013-726X

Corresponding author

Joe K. M. Fan, MBBS

Department of Surgery

Queen Mary Hospital

102 Pokfulam Road

Hong Kong

Fax: +852-28728425

drjoefan@hku.hk 